\title{
A review of BioFET's basic principles and materials for biomedical applications
}

\author{
Daeun Sung ${ }^{1} \cdot$ Jahyun Koo ${ }^{1,2}$ (D) \\ Received: 19 January 2021 / Revised: 3 March 2021 / Accepted: 29 March 2021 / Published online: 9 April 2021 \\ (c) Korean Society of Medical and Biological Engineering 2021
}

\begin{abstract}
Interest in biomolecular sensors for diagnosis of early diseases and prognosis of the diseases is increasing day by day. Among them, FET-based sensors are very useful in that of their versatile operating characteristics using various materials. Herein, after addressing the basic principles of BioFET, we conduct an overall review of BioFET on two of the main structural elements: transducing materials and probes. Transducing materials were classified into graphene, carbon nanotube, silicon, MOF, etc., and probes were classified into antibodies, enzymes, aptamers, etc.. The important elements in designing BioFETs, such as electrical properties of each material, Debye length, and fabrication process are introduced along with their respective structures and materials. After the review of each of these structures and characteristics, examples are discussed along with sensitivity, selectivity, and limit of detection. In addition to the operating aspects of the senser, novel processes, treatments, and materials that can be considered for various purposes are also introduced. Based on the understanding, an overview of diverse examples is given by dividing the applications of BioFET into three main types: antigen sensing, biomarker sensing, and drug effect monitoring. Focusing on these general reviews, we conclude how the future direction of development will move forward and what the main challenge is.
\end{abstract}

\section{Introduction}

The recent virus disease and the pandemic situation have sparked interest in versatile types of sensors, such as optical, electrochemical, and resonant biosensors. They can alert the patient's dangerous conditions or provide daily monitored information. The biosensors have key features that should be achieved: almost real-time response, stability of sensor characteristics, and sensing of low concentrated molecules, etc. To satisfy the various requirements, many researchers have studied transistor-based sensors, called BioFETs, which originated from electronic engineering field in 1970 [1]. Due to its enhanced sensitivity and selectivity as well as ability to be easily functionalized, BioFET began to be in the limelight in early-diagnosis, drug screening, and disease screening fields.

Jahyun Koo

jahyunkoo@korea.ac.kr

1 School of Biomedical Engineering, Korea University, Seoul 02841, Republic of Korea

2 Interdisciplinary Program in Precision Public Health, Korea University, Seoul 02841, Republic of Korea
Field effect transistor (FET) is an essential electronic component to compose an active device fabricated with combinations of gate, drain, and source. The source and drain are the electrodes which are the starting and arrival point of the charge carrier, and the gate is the connecting part of those. Namely, charge carriers (electrons in n-type FET, holes in p-type FET) flow from source to drain through gate, whose number can be increased or decreased by the voltage between a gate and source. Conventional FETs artificially alter the voltage applied to the gate by external power sources, while the voltage of BioFETs is changed by concentration and species of biomolecules that are chemically conjugated on the gate. The change of electrostatic charge environment or the charge transfer from the biomolecules themselves to the transducing nanomaterial induces the change in gate voltage $[2,3]$. This means that the amount of charge carrier that can cross the gate varies by concentration of proteins or antigens with specific charge values. That is, detection is achieved by measuring the change in conductance $\left(\Delta G / G_{0}\right)$ or the change in source-drain current $\left(\Delta I / I_{0}\right)$, or shift in the Dirac point. The change in conductance $\left(\Delta G / G_{0}\right)$ and the change in source-drain current $\left(\Delta I / I_{0}\right)$ is shown in response graphs of Fig. 2, and the shift of 
graphene's Dirac point after the detection of the target molecule is shown in Fig. 1a [4]. A typical example of the charge carrier itself moving to transducing nanomaterials is the already well-known glucose sensing mechanism. Shin et al. demonstrated highly sensitive glucose sensor which has $1 \mathrm{nM}$ of detection limit and major principles of BioFET [5]. Two electrons emerged by the oxidation-reduction (redox) reaction of palladium hydroxide $\left(\mathrm{Pd}(\mathrm{OH})_{2}\right)$ are transferred to the gate electrode, which in turn increases the density of the hole in the conducting channel. Apart from this, Fig. $1 \mathrm{~b}$ shows examples of various diseases and biomarkers that BioFET can be used as diagnostic of monitoring sensors [5-8]. To sum up, the BioFETs can have high sensitivity and selectivity without any labeling and high-cost production. In addition, the BioFETs do not need bulky equipment for measurements and provide cost-efficient, portable, convenient, personalized, and daily diagnosis [9]. For instance, BioFET can have sensitivity in the range of $\mathrm{fM}$ to aM without labeling, unlike electrochemical sensors that require labeling such as fluorescence and nanoparticles to prevent cross-reaction [10]. Here, we discuss the various materials and probes of BioFETs that determine sensitivity and selectivity, which are the key characteristics of BioFETs. Then, we introduce recent representative biomedical applications.

\section{Main}

\subsection{Carbon-based transducing materials, to utilize outstanding semiconducting property}

Carbon-based materials derived from a specific arrangement of carbon atoms have been attractive transducing materials due to their unique properties and nanostructures: controlled functionalization due to the carbon atoms, effective singlemolecule detecting due to the same size as a target molecule, as well as high conductivity, and chemical stability [11]. a
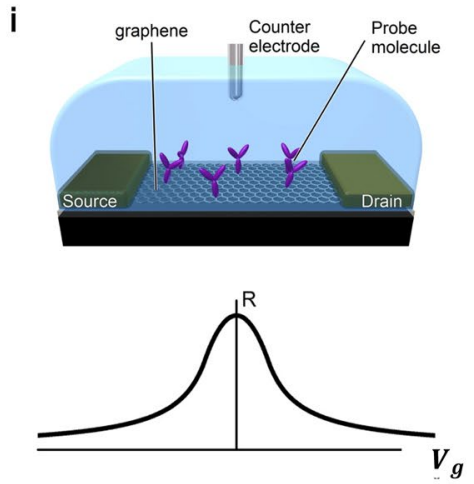

ii

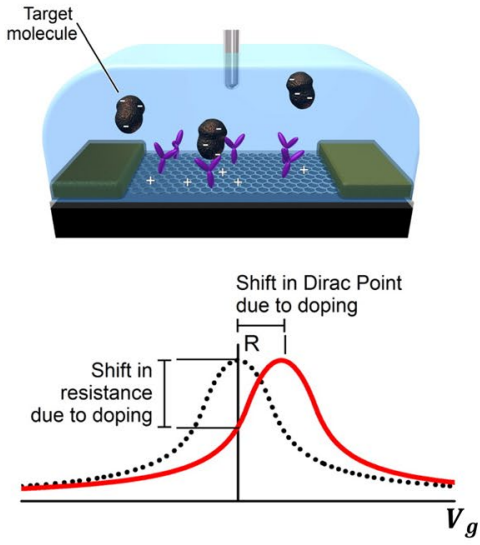

b

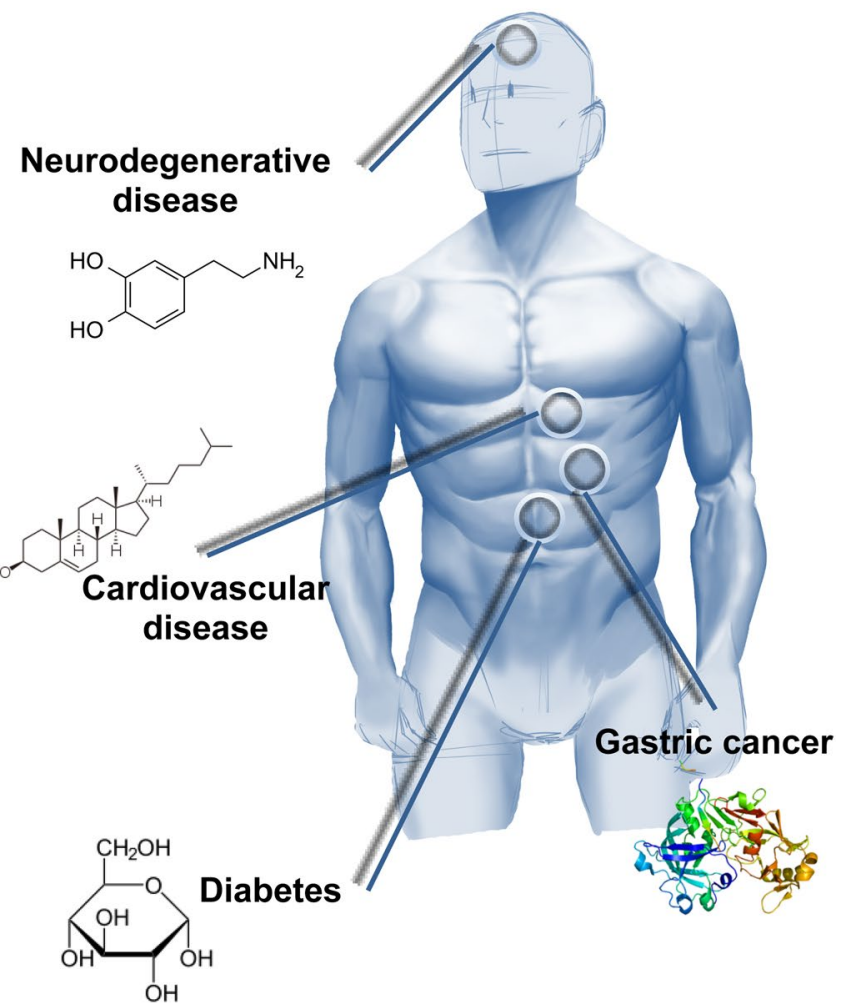

Fig. 1 The operating principles of BioFET using graphene and its applicability. a The illustration and the response graph when the target molecule does not exist in the sample (i). The illustration and the shift of response graph when the charge of the target molecule affects the distribution of charge of graphene through bonding reaction (ii). (Reproduced with permission, 2013, ACS). b Representative diseases that may occur in each body organ and their biomarkers that can be used to screen them. The structures of dopamine, cholesterol, cathepsin E (CatE), and glucose are shown, which are the biomarkers of neurodegenerative disease, cardiovascular disease, gastric cancer, and diabetes in order. They have been successfully detected by the sensor using the principle of BioFET [5-8] 
CNTs are one-dimensional nanostructures created through $\mathrm{C}-\mathrm{C} \mathrm{sp}{ }^{2}$ hybridization, one of the strongest bonds in nature. Only three of the four electrons in an atom are shared covalently with a conjugating carbon atom, and the left electron is delocalized. This is $\mathrm{sp}^{2}$ hybridization, and when hexagonal lattices of carbon atoms create a curved surface, it grows as a tube structure, CNT [12]. Due to their anisotropic structure, carbon nanotubes have a variety of electronic properties depending on their diameter or helicity. For example, the larger the diameter of the tube, the closer the bandgap converges to zero [13]. Such an example implies that we can increase the conductivity of CNT, resulting in high sensitivity of the biosensor. Like the delocalized electrons giving CNT's conductivity, the high aspect ratio of CNT allows efficient surface modification or functionalization, which enables to detect low concentration molecules and fast response time in sensor [14].

Graphene has similar characteristics to those of CNT because their similarity in the delocalized $\pi$-bond in $\mathrm{sp}^{2}$ hybridization structure provides high conductivity and mobility as in CNT. The graphene, which changes the band gap and electronic properties sensitively according to the number of layers in the stack, also has an ambipolar electrical field effect [13]. This implies that the graphene can also be used for BioFETs that sense through changes in the Dirac point. Aside from the mentioned characteristics of the CNT and the graphene, these two unique carbon nanostructures have the advantage of having a substantial surface/volume ratio, which can provide more active sites and increase sensitivity. For example, intentionally reduced charge screening by deforming the $2 \mathrm{D}$ graphene into a $3 \mathrm{D}$ structure contributed to detect 18,600 nucleic acid molecules in buffer and human serum samples: $600 \mathrm{zM}$ and $20 \mathrm{aM}$, respectively [15]. These nanostructured carbon materials enable high sensitivity and fast response time, which is essential for accurate, personalized, and prompt diagnosis in the clinical use of sensors, as shown in Fig. 1b [16].

Besides their physical-chemical properties, the carbon structures demonstrate suitable and unique properties as a component of BioFETs: reduced densification-defects due to well-established, simple manufacturing processes and easy functionalization. Like carbon-based nanodiamond (ND) and fullerene (FLN) structures are used in several sensors, other carbonaceous structures can be utilized in BioFET, taking advantage of these. For example, the electrochemical sensors using ND have high surface activity, high mechanical stability, functionality, and adaptability due to the dopants and unsaturated chemical bonds [17]. Electrostatic background noise, defects from the functionalization, and difficulty in determining optimal surface properties are considered as a critical challenge [18].

The followings are representative examples of BioFETs using carbon-based nanostructures.
Figure 2a shows CA125 (ovarian cancer antigen) FET sensor and SARS-CoV-2 virus FET sensor, each using graphene and its derivative, reduced graphene oxide (rGO) [19, 20]. In CA125 FET (Fig. 2a(i)), carboxylated-MWCNT (MWCNTs$\mathrm{COOH}$ ) was modified with ssDNA, which can be bound to CA125 antigen. This CNT-aptamer probe was put on the PMMA substrate with sputtered Au electrodes and eventually showed decreasing $I_{d}$ with increasing target molecule concentrations $\left(\mathrm{V}_{\mathrm{d}}=0.2 \mathrm{~V}\right.$ and $\mathrm{V}_{\mathrm{g}}=0.0-1.0 \mathrm{~V}$, concentrations ranging from $1.0 \mathrm{E}-9 \mathrm{U} / \mathrm{mL}$ to $1.0 \mathrm{U} / \mathrm{mL}$ ). The negative charge of CA125 antigen ( $\mathrm{pI}$ of 7.3) in PBS (pH 7.4) leads to the depletion of the n-type rGO channel when a binding event occurs, resulting in the reduction of $\mathrm{I}_{\mathrm{d}}$. Another SARS-CoV-2 example is shown in Fig. 2a (ii). With antibody, the SARS-CoV-2 virus can be directly detected through its spike protein. The SARSCoV-2 virus FET sensor, which used the attached antibody on graphene sheet using PBASE, showed a real-time response to at least $1 \mathrm{fg} / \mathrm{mL}$ of antigen in PBS and also to the clinical samples collected by nasopharyngeal swabs (stored in UTM).

The second example of transducing carbon nanomaterial, CNT-used BioFETs, is also given in Fig. $2 \mathrm{~b}$ and proposes various applications of CNT-BioFET [21, 22]. In Fig. 2b (i), diagram of one of the CNT floating gate FET (CNT FG-FET) array is introduced. Ultrathin $\mathrm{Y}_{2} \mathrm{O}_{3}$ layer as high- $\kappa$ dielectric material was put onto the usual CNT channel by deposition followed by the immobilization of probe DNA. Due to the $\mathrm{Y}_{2} \mathrm{O}_{3}$ layer, the channel of FET can be isolated from the sample solution, therefore interactions other than hybridization with target DNA do not affect $\mathrm{I}_{\mathrm{d}}$. In other words, CNT was passivated to refine the signal. There is a different kind of example that probe on the CNT was uniquely formed in Fig. 2b (i). So-called 'nanodisc-functionalized bioelectronic nose (ND-ONBN)' was developed by embedding nanodiscs with an olfactory receptor to detect cadaverine (CV) target molecule. FET using CNT and Pd/Au electrodes was fabricated, and with half- $\mathrm{V} 5 \mathrm{Ab}$ (antibody), olfactory receptor TAAR13c-embedded nanodisc (T13NDs) was successfully immobilized on electrodes on CNT. Here, it can be also said that half-V5 Ab was used to immobilize T13NDs in an desired orientation via their thiol groups because of the TAAR13c's V5 epitope. The real-time response of an ONBN device in terms of conductance depending on various concentrations of $\mathrm{CV}\left(\mathrm{V}_{\mathrm{ds}}=0.1 \mathrm{~V}\right.$, adding $\mathrm{CV}$ solution to HEPES buffer) is on the right. As we can see from the graph, the ONBN device could detect at least $10 \mathrm{pM}$ of $\mathrm{CV}$. 
$\mathbf{a}$

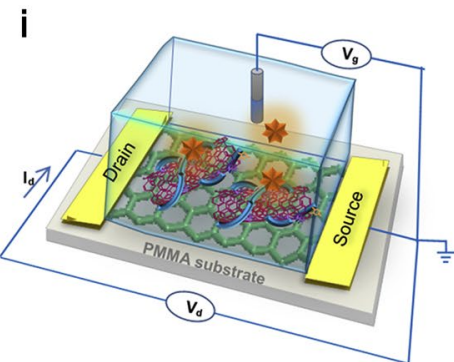

b

i

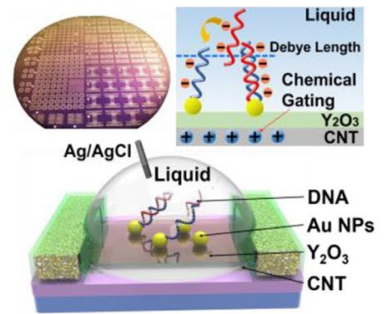

ii

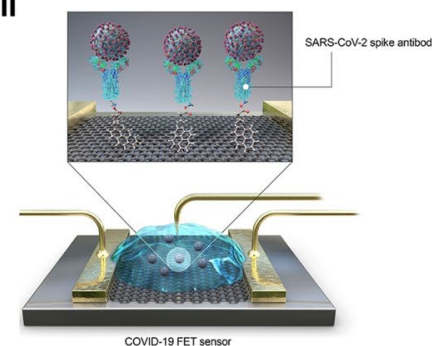

ii

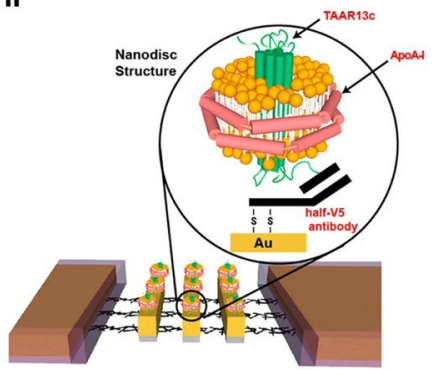

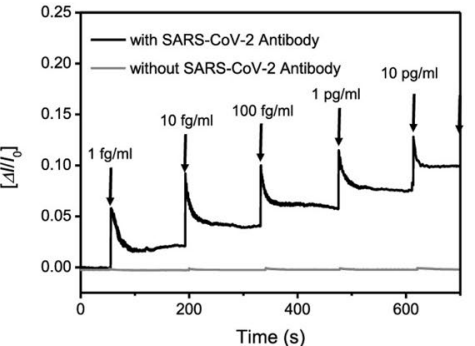

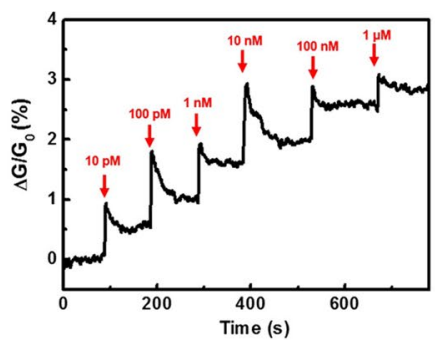

Fig. 2 Carbon nanomaterial in BioFET: sensors using graphene, CNT as transducing material. a Illustration of CA125 (ovarian cancer antigen) FET sensor built on reduced graphene oxide (rGO) (left) and SARS-CoV-2 virus FET sensor using graphene sheet as transducing material (middle). Each used rGO and graphene, the latter presenting a real-time response to $1 \mathrm{fg} / \mathrm{mL}$ of SARS-CoV- 2 antigen protein in PBS due to graphene's electron configuration (right). (Reproduced

\section{BioFET using other materials: silicon, inorganic compounds, metal-organic framework}

Other materials as transducing material consist of silicon and metal compounds, which can be fabricated by the specific fabrication processes. The fabrication processes of BioFETs can allow cost-effective and customized sensor platform for biomedical applications. For example, silicon deposition using e-beam lithography and chemical vapor deposition can build H1N1 virus and uranyl FET sensors, respectively $[23,24]$. The study of fabrication of silicon nanowire ( $\mathrm{SiNW})$ as transducing nanomaterial that enables label free, ultrasensitive, real-time detection is also actively underway. SiNW can be used for sensing various proteins, small molecules, nucleic acid, and viruses.

One of the most popular manufacturing methods for noncarbon materials is nozzle-jet printing. Nozzle-jet printing does not require masks and chemical etchants to fabricate source, drain electrodes, and gate parts [25]. This printing method is widely used, for instance, to create RFID tags using roll-to-roll (R2R) printing or to fabricate flexible displays due to its cost-effective and fast process [26, 27]. In another experiment, $\mathrm{ZnO}$ quantum dots precursor ink was added to solvent (methanol, ethanol, ethylene glycol, with permission, 2018, ELSEVIER and 2020, ACS). b Illustration of floating gate (FG) CNT FET sensor and nanodisc (ND) - functionalized bioelectronic nose (ONBN). Both combined specific materials, $\mathrm{Y}_{2} \mathrm{O}_{2}$ dielectric layer with floating gate (FG) and olfactory receptor with nanodisc each. ND functionalized ONBN shows real-time responses to $10 \mathrm{pM}$ of $\mathrm{CV}$ solution with various concentrations (right). (Reproduced with permission, 2020, ACS and 2017, ACS)

ethanolamine mixed with 4:16:1:1 volume ratio) [25]. Later, to grow $\mathrm{ZnO}$ nanorods ( $\mathrm{ZnO} \mathrm{NRs}$ ), $\mathrm{ZnO}$ seed substrate fabricated by nozzle-jet method was suspended upside down to a solution containing $\mathrm{Zn}$ to grow $\mathrm{ZnO}$ NRs. Just as graphenes and CNTs show electrical properties that depend on the number of layers and walls, silicon or inorganic compounds are characterized and advantageous by many different fabrication processes that vary their structure to contribute to more robust immobilization of probes and enhanced sensitivity. For example, nanowires (NWs) are more employed than their film analogs due to the unidirectional conduction and large surface-to-volume ratio [28]. Non-carbon materials with more free fabrication methods will continue to be more suitable for BioFET through various structures (Table 1).

Herein, we classified non-carbon nanomaterials into three categories: silicon, inorganic compound, and MOF. In Fig. 3a, there are two silicon-based nanostructures, siliconnanonet and SiNWs [23, 24]. Left upper optical and SEM image present structure of silicon-nanonet FET, fabricated by electron-beam lithography and ICP-RIE of silicon-oninsulator (SOI) wafer, followed by thermal oxidation as insulator making process. Monoclonal antibody $(\mathrm{mAb})$ that binds to influenza A (H1N1) virus was immobilized onto silicon-nanonet structure by APTES and glutaraldehyde treatment. Bovine serum albumin (BSA) was used to prevent 
Table 1 Representative three types of FET's applications and their organized examples based on their transducers, probes, etc

\begin{tabular}{|c|c|c|c|c|c|c|}
\hline Application & Target & Transducing material & Probe & Used sample & LoD & Ref \\
\hline \multirow[t]{7}{*}{ Antigen sensing } & $\begin{array}{l}\text { Ovarian cancer anti- } \\
\text { gen (CA125) }\end{array}$ & Graphene (GO) & Aptamer & Serum & $5 \mathrm{E}-10 \mathrm{U} / \mathrm{mL}$ & [19] \\
\hline & $\begin{array}{l}\text { SARS-CoV-2 virus } \\
\text { spike protein }\end{array}$ & Graphene & Antibody & $\begin{array}{l}\text { Nasopharyngeal } \\
\text { swab (PGR) }\end{array}$ & $\begin{array}{l}1 \mathrm{fg} / \mathrm{mL}(\mathrm{PBS}) \\
100 \mathrm{fg} / \mathrm{mL} \text { (clini- } \\
\text { cal) }\end{array}$ & [20] \\
\hline & $\begin{array}{l}\text { Influenza A (H1N1) } \\
\text { virus }\end{array}$ & Silicon & Antibody & PBS & $10 \mathrm{pg} / \mathrm{mL}$ & [23] \\
\hline & PSA protein & Silicon & Antibody & Serum (diluted) & $1 \mathrm{ng} / \mathrm{mL}$ & [31] \\
\hline & Hemolytic toxins & CNT & $\begin{array}{l}\text { RBC membrane } \\
\text { vesicle }\end{array}$ & PBS & $\begin{array}{l}\text { fM range (varied by } \\
\text { type of toxin) }\end{array}$ & [36] \\
\hline & $\begin{array}{l}\text { AIV virus nucleopro- } \\
\text { tein (AIV NP) }\end{array}$ & $\mathrm{SnO}_{2}$ & Antibody & $\begin{array}{l}\text { Live animal cloacal } \\
\text { swab }\end{array}$ & $10^{3} \mathrm{EID}_{50} / \mathrm{mL}$ & [45] \\
\hline & $\begin{array}{l}\text { Evola virus glycopro- } \\
\text { tein (EGP) }\end{array}$ & Graphene (RGO) & Antibody & PBS, serum, plasma & $1 \mathrm{ng} / \mathrm{mL}$ & [46] \\
\hline \multirow[t]{3}{*}{ Biomarker sensing } & $\begin{array}{l}\text { AFP (alpha-fetopro- } \\
\text { tein) }\end{array}$ & Silicon & Antibody & Mice serum & $50 \mathrm{fg} / \mathrm{mL}$ & [32] \\
\hline & $\mathrm{ACh}$ & Graphene (RGO) & Enzyme & PBS Cultured cell & $1 \mu \mathrm{M}$ & [34] \\
\hline & $\mathrm{A} \beta 42$ & Graphene (GO) & Aptamer & PBS & $1 \mathrm{pg} / \mathrm{mL}$ & [47] \\
\hline \multirow[t]{2}{*}{$\begin{array}{l}\text { Monitoring drug } \\
\text { effect }\end{array}$} & TNF- $\alpha$ & CNT & Antibody & $\begin{array}{l}\text { Cultured cell media } \\
\text { (raw } 264.7 \text { cell) }\end{array}$ & $1 \mathrm{pg} / \mathrm{L}$ & [48] \\
\hline & $\begin{array}{l}\text { Dopamine released } \\
\text { by cell }\end{array}$ & CNT & ABTS radical & $\begin{array}{l}\text { Cultured cell solution } \\
\text { (PC12 cell) }\end{array}$ & $10 \mathrm{nM}$ & [49] \\
\hline
\end{tabular}

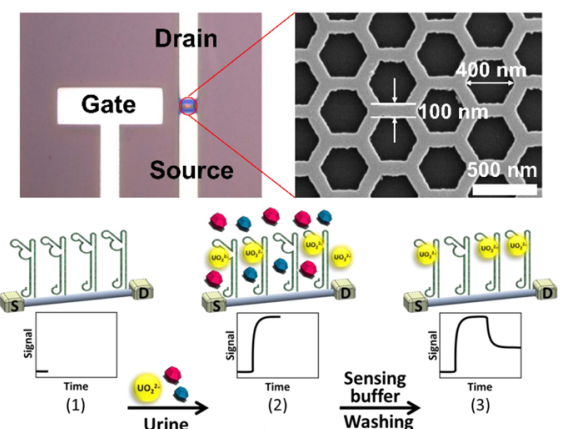

b

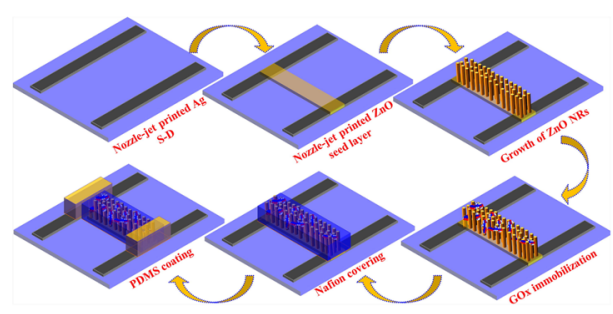

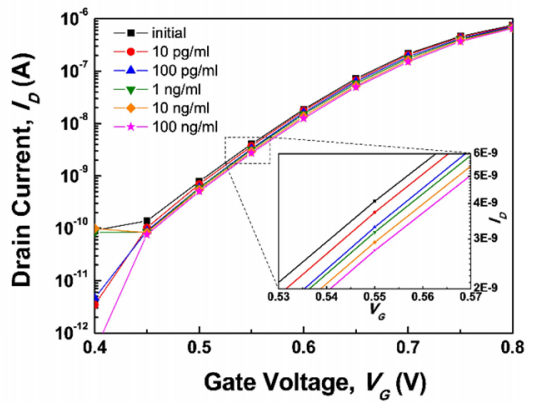

c

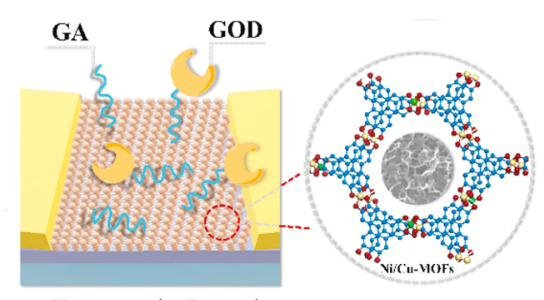

Enzymatic Reaction
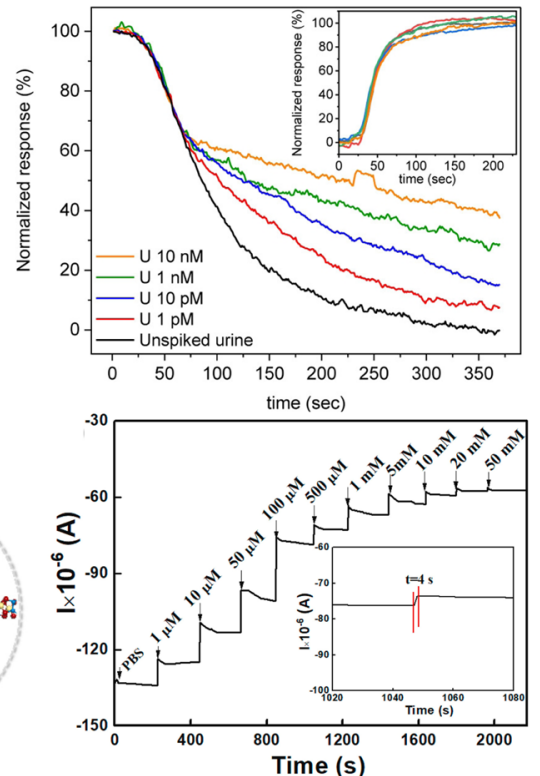

Fig. 3 Silicon, inorganic compound, MOF as transducing material: capable of being diverse structures. a Optical and SEM images of silicon-nanonet used in influenza A (H1N1) virus FET sensor (left, upper), an illustration of SiNW-FET biosensor modified with uranyl-binding aptamer (left, lower). Transfer curve with various concentrations showed LoD of $10 \mathrm{pg} / \mathrm{mL}$ in H1N1 PBS solution (middle). The graph on the far right shows a normalized response to spiked, undiluted urine samples as a function of time. (Reproduced with permission, 2019, IEEE and 2020, ACS). b, Schematic illustra- tion of fabricating nozzle-jet printed glucose FET sensor. Nozzle-jet printing of silver electrodes and $\mathrm{ZnO}$ nanorod ( $\mathrm{ZnO} \mathrm{NR}$ ) followed by glucose oxidase (GOx) immobilization, achieving highly reproducible enhanced sensing performance. (Reproduced with permission, 2017, ELSEVIER). c, Illustration of Ni/Cu-MOFs-FET ( $\mathrm{Ni}-\mathrm{Cu}$ metal-organic framework (7:1) on FET). Response curve in the range of $1 \mu \mathrm{M}$ to $50 \mathrm{mM}$ glucose was also given with inset data $(100 \mu \mathrm{M})$, which shows response time less than $5 \mathrm{~s}$. (Reproduced with permission, 2021, ELSEVIER) 
non-specific binding to the unreacted active sites. The silicon-nanonet FET showed shift of response curve: as the concentration of virus in PBS (pH 7.4) increases, response curve shifts laterally to the right (Fig. 3a, middle). This is due to the negative charge of the $\mathrm{H} 1 \mathrm{~N} 1$ virus $(\mathrm{pI}=5.4)$ in PBS ( $\mathrm{pH}$ 7.4), meaning that electrons in the channel are repelled and resulted in the reduction of $\mathrm{I}_{\mathrm{d}}$.

Left lower illustration shows uranyl ions-detecting $\mathrm{Si}$ NW-FET. This FET sensor, intended to measure the amount of uranium that may be caused by exposure to various industrial environment, succeeded in sensing undiluted urine samples using SiNW. As shown in the figure, the uranyl-binding aptamer was immobilized to SiNW, which was synthesized using a chemical vapor deposition [29]. The method of amino-silane modification of SiNW (inducing the surface interaction with silanized SiNW and carboxy-terminated aptamers) was used to immobilize the aptamer. The SiNWFET array was also assembled with polydimethylsiloxane (PDMS) channel to expedite the sensing of urine. The measurement was done with washing away by buffer solution, showing the detection limit in $\mathrm{pM}$ units in a sample of 15 $\mu \mathrm{L} / \mathrm{min}$.

Figure $3 b$, $c$ show the examples of BioFETs using $\mathrm{ZnO}$ nanorods (ZnO NRs) and Ni-Cu MOF (Ni-Cu metal-organic framework (7:1)) as transducing material, respectively [25, 30]. The vertical $\mathrm{ZnO}$ NRs fabricated by the growing process mentioned above have high reproducibility, enzyme loading, and sensitivity. Glucose oxidase (GOx) immobilization through Nafion coating helped reducing interference, resulting in a limit of detection of $0.07 \mathrm{mM}$ (100 mM PBS, $\mathrm{pH}$ 7.4). When using actual human serum samples, they showed about a $15 \%$ decrease in sensor response because of small molecules interfering the spread of glucose. The $\mathrm{Ni} / \mathrm{Cu}-\mathrm{MOF}$-FETs found in Fig. $3 \mathrm{c}$ are the sensors that utilize the widely used enzyme-using principle to sense glucose. The enzyme (glucose oxidase) connected to the MOF via glutaraldehyde showed a detection limit of $0.51 \mu \mathrm{M}$, which is lower than the previous case. $\mathrm{NiCl}_{2} \cdot 6 \mathrm{H}_{2} \mathrm{O}$ and
$\mathrm{CuSO}_{4} \cdot 5 \mathrm{H}_{2} \mathrm{O}$ were used to mix $\mathrm{Ni}$ ions and $\mathrm{Cu}$ ions into DI water at the appropriate molar rate and then mixed with other substances in ligand solution to produce MOF. These MOF-FETs had a short response time of less than $5 \mathrm{~s}$ due to their high reactivity (Table 2 ).

\section{Various types of BioFET probes for sensitivity and adequate response time: antibody, enzyme, aptamer, etc}

The types of BioFET's probes, which directly bind to the target molecules, are key indicators to provide adequate sensitivity and signal response time. Various types of probes, such as antibody [31, 32], enzyme [33, 34], aptamer [19, 35], and others [36, 37], can be used in practice. However, determining the probe requires an understanding of the concept of Debye length, which is related to the charge screening effect in samples. The concept of Debye length limits the choice of probes, thus it is highly related to the BioFET's sensitivity.

Debye length occurs when the charge of biomolecules, which act to change the voltage of the gate in BioFET, is screened. For instance, molecules with negative charges, such as DNA, will be surrounded by molecules with positive charges in a solution. In this case, there will be a specific distance at which the numbers of positive and negative charges are equal. This is called Debye length $\left(\lambda_{D}\right)$ and can be expressed as: $\lambda_{D}=\frac{1}{\sqrt{4 \pi l_{B} \sum_{i} \rho_{i} z_{i}^{2}}}$ [38]. (where $l_{B}=0.7 \mathrm{~nm}$ (Bjerrum length at room temperature), $\rho$ is the density and $z$ is the valence of each ions). This equation results in a value of 0.7 to $2.2 \mathrm{~nm}$ in a typical physiological sample, which is much smaller than the 5 to $10 \mathrm{~nm}$ length of an IgG antibody commonly used as a probe $[39,40]$. As a result, the charge of target molecules such as proteins or target DNAs is screened, making efficient sensing impossible [41]. This suggests that the size of the probe in BioFET can have a significant impact on sensitivity, so small probes like

Table 2 Other organized examples based on their transducers, probes, etc

\begin{tabular}{|c|c|c|c|c|c|c|}
\hline Application & Target & Transducing material & Probe & Used sample & LoD & Ref \\
\hline \multirow[t]{8}{*}{ others } & $\begin{array}{l}\text { Specific DNA } \\
\text { sequence \& } \\
\text { microvesicles }\end{array}$ & $\mathrm{CNT}$ (with $\mathrm{Y}_{2} \mathrm{O}_{3}$ ) & DNA & PBS & $\begin{array}{l}60 \text { aM, } 6 \text { parti- } \\
\text { cles } / \mu \mathrm{L} \text { (theoreti- } \\
\text { cally) }\end{array}$ & [21] \\
\hline & Cadaverine (CV) & CNT (with $\mathrm{Au}$ ) & Olfactory receptor & Real food & $10 \mathrm{pM}$ & [22] \\
\hline & Uranium & Silicon & Aptamer & Urine & $1 \mathrm{pM}$ & [24] \\
\hline & Glucose & $\mathrm{ZnO}$ nanorod ( $\mathrm{ZnO} \mathrm{NR})$ & Enzyme & PBS & $0.07 \mathrm{mM}$ & [25] \\
\hline & Glucose & $\mathrm{Ni} / \mathrm{Cu} \mathrm{MOF}$ & Enzyme & PBS & $1 \mu M$ & [30] \\
\hline & Glucose & $\mathrm{WSe}_{2}$ (plasma treated) & Enzyme & Glucose solution & $1 \mathrm{mM}$ & [33] \\
\hline & DNA & Graphene & DNA & PBS & $100 \mathrm{fM}$ & [35] \\
\hline & Oxalic acid (OA) & $\begin{array}{l}\text { None (probe immobilization } \\
\text { through covalent bond) }\end{array}$ & Pt_cPPyNFs & PBS & $10 \mathrm{fM}$ & [37] \\
\hline
\end{tabular}


aptamers are often used recently rather than large probes, such as antibodies.

Besides, post-treatments that immobilize probes to satisfy Debye length onto the transducing material should be followed. As in general electrochemical sensors, orientation of the probe molecule's fixation in BioFETs is critical in that the sensing part must directly attach to the target molecules in the sample solution. Surface modification and design, covalent attachment, (bio) affinity techniques can be chosen as a way to attach antibodies in the correct orientation [42]. The method of how to immobilize DNA is also introduced, which can be summarized simply as physical adherence, covalent bonding, and streptavidin-biotin interactions $[43,44]$. Among these methods, the most commonly used method in the field of BioFETs is covalent attachment (covalent bonding). 1-pyrenebutanoic acid, succinimidyl ester (PBASE), glutaraldehyde, (3-aminopropyl) triethoxysilane (APTES) are used to immobilize probes to the surface of the substrate. Especially, glutaraldehyde and APTES are often used to treat the surface of the substrate on which the probe is fixed. For example, the conductive nanostructures of the gate which the probe is to be immobilized were terminated with a hydroxyl $(-\mathrm{OH})$ group through ethanol treatment, and the hydroxyl group was replaced by amine $\left(-\mathrm{NH}_{2}\right)$ group and aldehyde (-CHO) group by APTES and glutaraldehyde in order [23]. The antibody was immobilized when the aldehyde group and the amine group of the antibody formed an amide bond. Such covalent anchoring can have the effect of increasing stability and reducing non-specific coupling in a biosensor [2].

Figure 4 shows several BioFETs, categorized by the probe used. In order, various probes such as antibodies [31, 32], enzymes [33, 34], and aptamers [19, 35] are shown, and additional methods for increasing selectivity are also proposed.

Figure 4a shows an example of antibodies being used as a probe [31,32]. Mirisan et al. measured the thickness of antibodies after their immobilization process (Fig. 4a (i)) so that the reproducibility of the sensor was not affected due to the problem with the Debye length. FE-SEM images were used to measure SiNW thickness after functionalization and calculate the relative standard deviation (RSD). Figure 4a (ii) shows the use of bovine serum albumin (BSA) to block the area of sensors where antibodies do not exist, which helps with the stability of sensing. Here, the Debye length was also calculated to ensure that the sensor has a high sensitivity. The calculation of Debye length in the defined experiment conditions $\left(0.1 \times \mathrm{PBS}, \lambda_{D} \approx 0.32 I^{-0.5}\right)$ obtained results of $2.4 \mathrm{~nm}$.

In Fig. 4b, we can see examples of enzymes used as a probe $[33,34]$. In the experiment of Fig. $4 \mathrm{~b}$ (i), $\mathrm{O}_{2}$ plasma treatment has increased the number of areas where the probe linker APTES binds, effectively immobilizing the
GOx, which is the commonly used enzyme in BioFETs. The fact that reusable BioFETs are created by the reusability of GOx suggests that materials and fabrication methods of BioFET have a significant impact on device operation. Figure $4 \mathrm{~b}$ (ii) also shows that BioFET can be used for novel things, depending on the characteristics of the probe. To detect acetylcholine (ACh), the acetylcholinesterase (AChE) molecule was immobilized to RGO using PBASE, and therefore studies related to enzyme inhibitors were possible. After the topographic analysis method being used to check immobilization, the resulting BioFET obtained results that could be used to evaluate enzyme inhibition reaction, especially using AChE inhibitor called donepezil and libastigmine.

An example of how aptamer or DNA can be used as a probe is shown in Fig. 4c [19, 35]. S. Mansouri Majd et al. conducted an experiment like Fig. 4c (i), where the multiwalled carbon nanotubes (MWCNT) and aptamer were combined as probes. MWCNT's carboxylic acid group, processed by EDC-NHS, formed an amide bond with the amine group of aptamer, allowing the binding to take place within the Debye length. This resulted in improved sensitivity and therefore succeeded in sensing ovarian cancer antigen (CA125) in the human serum samples. Figure 4c (ii) shows a typical method of detection through DNA hybridization. When DNA hybridization occurs, the $\mathrm{I}_{\mathrm{ds}}-\mathrm{V}_{\mathrm{gs}}$ graph is shifted to the positive $\mathrm{V}_{\mathrm{g}}$ direction, as the combination with full comprehensive (FC) target DNA leads to negative potential gating in the graphene. The shift of charge neutrality point voltage $\left(\Delta \mathrm{V}_{\mathrm{cnp}}\right)$ was described a s : $\Delta V_{c n p}=\Delta V_{\max }\left(1-e^{-\left(k_{o n}[A]+k_{o f f}\right) t}\right)($ adsorption $), \Delta V_{c n p}=$ $\Delta V_{\max } e^{-k_{\text {off }} t}$ (desorption). (where $\Delta V_{\max }$ is the maximum response signal at [A] of DNA concentration. $\mathrm{k}_{\text {on }}$ and $\mathrm{k}_{\text {off }}$ are the rate constants of association and dissociation.).

Finally, in Figs. 4d and e, a slightly unusual probe material is introduced [36, 37]. By coating the cell membrane of red blood cells on the CNT like in Fig. 4d, various types of pore-forming hemolytic toxin could be adsorbed, such as melittin, streptolysin $\mathrm{O}$, and alpha hemolysin. They changed the BioFET's channel conductance regardless of their structural or chemical properties when they formed toxin-biomembrane interaction. It is an example of the ability to design biomimetic BioFETs by using creative probes and typical probes. Unlike Fig. 4d, which uses bio-derived probes, Fig. 4e uses Pt nanoparticles to sense oxalic acid (OA), which causes kidney disease. The electro-oxidation of OA molecules was caused by the catalytic effect of $\mathrm{Pt}$ nanoparticles immobilized on polypyrrole-3-carboxylated nanofiber (Pt_cPPyNF), which successfully overcame the instability of conventional enzyme-based OA sensors. The Pt_cPPyNFs formed by $\mathrm{PtCl}_{4}$ and $\mathrm{NaBH}_{4}$ demonstrated the sensitivity of $10 \mathrm{pM}$, which demonstrates the potential of $\mathrm{Pt} \_\mathrm{cPPyNF}$ as various probes. 
a

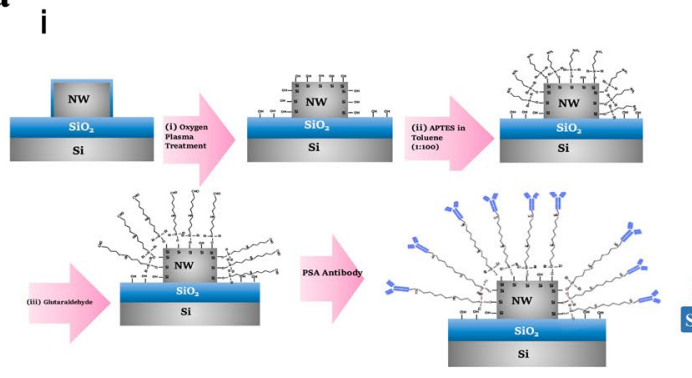

b

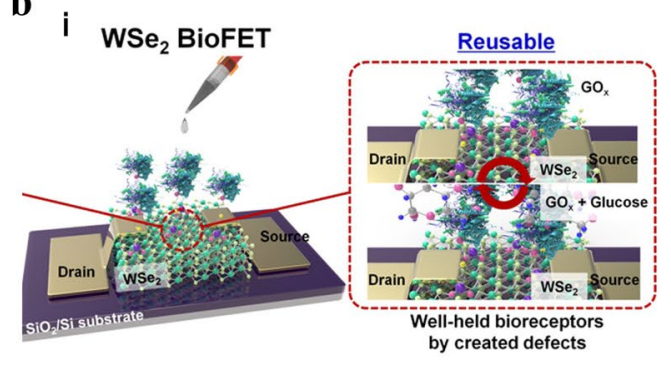

c

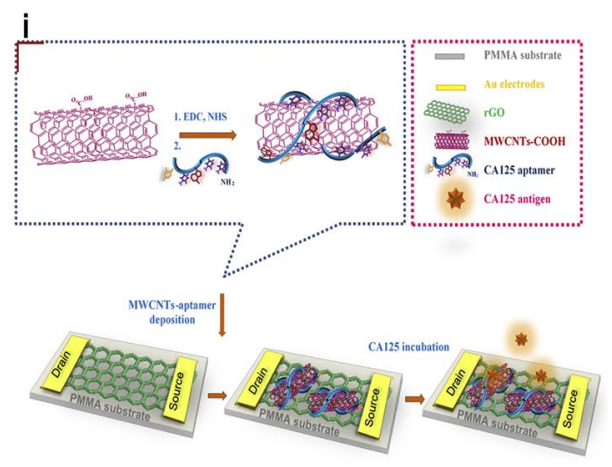

d

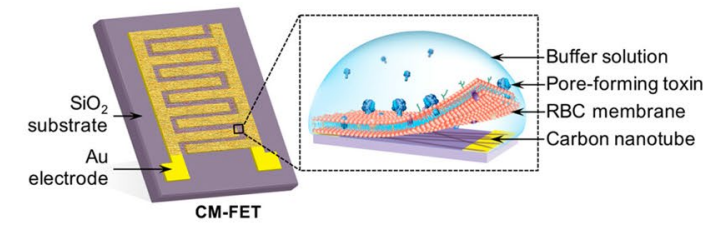

ii

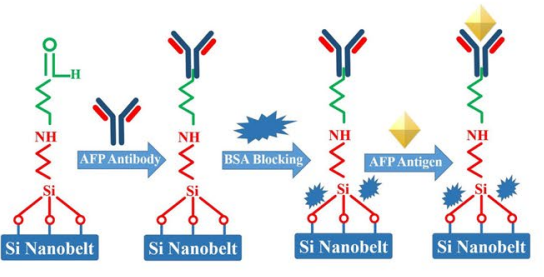

ii Enzymatic reaction Enzyme inhibition
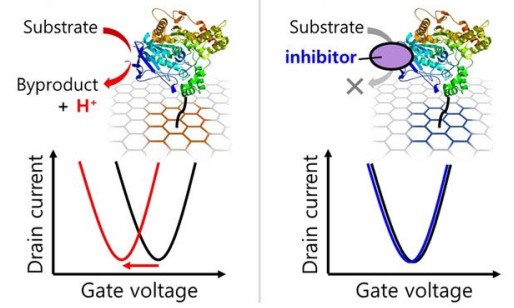

ii

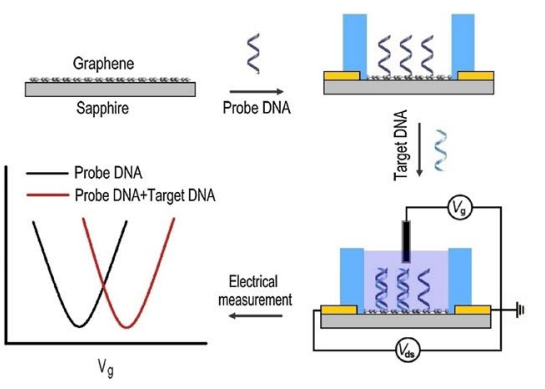

$\mathbf{e}$
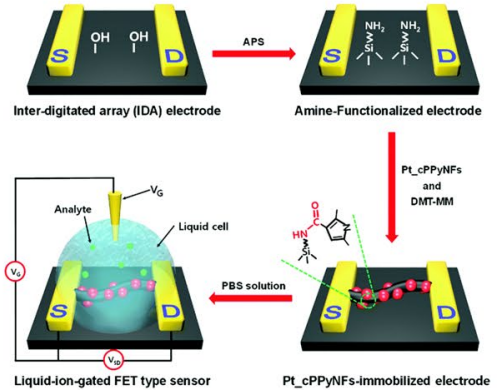

\section{Representative biomedical applications using BioFET}

So far, we have looked at several types of materials of BioFET's structures and concepts associated with them. The range of these BioFET's applications is extensive, and the representative examples of applications will be divided into three categories here. BioFETs for detecting antigens (especially viruses) [45, 46], BioFETs for detecting biomarkers of certain diseases [47], and BioFETs for drug screening [48, 49] are demonstrated in Fig. 5a-c, respectively.
Figure 5a (i) and Fig. 5a (ii) show BioFETs that allow the antigen to be sensed by the proteins that structure the viruses $[45,46]$. FET sensor, which has a response graph in Fig. 5a (i), is the BioFET that detects nucleoproteins (NP) of avian influenza viruses (AIVs). NPs are the structural proteins inside the virus that had relatively few subtypemutations, thus facilitating the detection and the point-ofcare of the AIVs. The sensing of NPs of AIV was carried out by the tendency that the negative voltage shift increases depending on the concentration of the viruses. Figure 5a (ii) is the response graph of the BioFET that is for detecting 
४Fig. 4 Various types of BioFET probes: antibody, enzyme, aptamer, etc. a Several antibody immobilization processes. The functionalization process of silicon nanowire (SiNW) included an etching of SiNW for oxide elimination, silanization with (3-Aminopropyl) triethoxysilane (APTES) initiator, and activation with glutaraldehyde (GA), which is for attaching antibodies. The illustration on the right shows how unreacted molecules are blocked for better sensitivity by bovine serum albumin (BSA). (Reproduced with permission, 2019, ELSEVIER and 2018, ELSEVIER). b, Principles of reusable $\mathrm{WSe}_{2}$ glucose-sensing BioFET and RGO-EnFET (enzyme-modified FET with reduced graphene oxide). Glucose oxidase was immobilized on $\mathrm{WSe}_{2}$, which attributed to the extra binding site and achieving high sensitivity (left). The feature that acetylcholinesterase (AChE)immobilized RGO-EnFET exhibited Dirac point shift $\left(\Delta V_{\text {Dirac }}\right)$ only without inhibitor allows them to be used in enzymatic kinetics field and drug screening field (right). (Reproduced with permission, 2018, ACS and 2018, ELSEVIER). c Examples of DNA / aptamerused FET sensor. They used aptamer attached to carboxylated multiwalled carbon nanotubes (MWCNTs) and probe DNA itself, which means DNA hybridization is the main principle of these FETs. Probe aptamer (ssDNA) and DNA were both immobilized through amine groups and can thus detect ovarian cancer antigen (CA125) and target DNA. (Reproduced with permission, 2020, ACS and 2018, ELSEVIER). d Structure of cell-membrane-coated CNT FETs (CM-FETs). By stacking the red blood cell (RBC) membrane onto FET, it can absorb and detect various hemolytic toxin as a biomimetic FET sensor. (Reproduced with permission, 2019, ACS). e, FET sensor, which used Pt decorated polypyrrole-3-carboxylated nanofibers (cPPyNFs) as a probe. Pt_cPPyNFs were fabricated by $\mathrm{PtCl}_{4}, \mathrm{NaBH}_{4}$ treatment with sonication, making OA molecules electro-oxidize via Pt nanoparticle's catalytic effect. (Reproduced with permission, 2018, Royal Society of Chemistry)

glycoproteins (GPs) of the Ebola virus. The glycoprotein (GP) $\mathrm{GP}_{1,2}$ present on the surface of all species of the Ebola virus, and the virus attachments to host cells occur through its lectin binding, followed by the secretion of sGP. This causal relationship between the presence of viruses and the secretion of sGP enabled direct detection of the viruses. Both the NP of AIV and the GP of the Ebola virus had the protein properties and could be used for direct antigen detection.

Biomarker detection of certain diseases, the second representative application, can be found in Fig. 5b [47]. The phenomenon that some misfolded proteins such as amyloidbeta $(\mathrm{A} \beta)$ deposits in the brain and eventually form a plaque in the central neural system is said to be associated with the Alzheimer's disease (AD). This led to the use of A $\beta 42$ for AD early diagnosis. The production of anti- A $\beta$ RNA aptamer using SELEX techniques allowed detection by inducing a shift of the response graph like Fig. $5 \mathrm{~b}$.

In the last Fig. 5c (i), we can confirm that BioFET can be used in the drug screening field [48, 49]. FET sensor determined that the concentration of tumor necrosis factor $\alpha$ (TNF- $\alpha$ ), secreted when lipopolysaccharides (LPS) were treated at Raw 264.7 cells, was decreased as the concentration of Lupeol increased. This means that the FET sensor can be used to assess the effectiveness of anti-TNF agents (Lupeol), which is critical to the treatment of inflammatory bowel disease (IBD) especially when it is considered that TNF- $\alpha$ is a pro-inflammatory cytokine associated with IBD. This BioFET, which enables to selectively detect TNF- $\alpha$ among various types of cytokines, can be said to be effective in drug screening. To the right of Fig. $5 \mathrm{c}$ (i), a similar principle of BioFET can be found. Similarly, the secretion of dopamine was promoted at PC12 cells by $K^{+}$, and the concentration of dopamine was measured as the concentration of Pimozide decreased. Dysfunctions in the dopamine secretion can induce fatal neurological diseases such as Parkinson's disease and schizophrenia. Thus, an antipsychotic drug helps treat neurological diseases by primarily controlling dopamine activity, therefore it can be said that this FET sensor is useful in drug screening of Pimozide-like drug. Figure 5a (ii) shows how each sensor responses to respective drugs and the sensing qualities. In other words, the application of BioFET can vary depending on the characteristics of the target being sensed by BioFET, so the understanding of the target molecules is also critical to the design of BioFET.

\section{Conclusions}

BioFET, which allows probes such as antibodies and aptamers to be fixed to graphene, CNT, MOF, etc., demonstrates a variety of applications. Efforts are being made to utilize the unusual structures of the BioFET, such as the 'Cell-based BioFET' and the 'Beetle/chip FET', which utilizes cell attaching to the gate insulator and an insect antenna with an olfactory receptor for sensing, respectively [1]. In addition to the material aspect, there are also studies in terms of signal processing such as quantitatively analyzing signal-to-noise ratio (SNR) to improve BioFET's performance [50]. Along with the studies about various materials, processing and analysis methods, the development of BioFET is endless. For instance, in the future direction of BioFET, the followings are also expected: the integration in the micro total analysis system ( $\mu$-TAS) or lab on a chip system, biomolecules-based FET directly utilizing the bio-derivatives [51]. Furthermore, enhancement in higher sensitivity and reproducible operations will open up a new future-diagnostic paradigm, as they are the main obstacles to the commercialization of BioFET sensors [9]. For example, the body fluids can be utilized for early diagnosis of cancer and identification of virus infections, or monitoring the prognosis of the disease continuously. Just as advances in biomedical fields have evolved through convergent collaboration in various fields, BioFET also needs an integrated view of biotechnology, semiconductor engineering, material engineering, electronics, microprocesses, etc. This effort will help overcome the biggest challenge of the current BioFET technologies: A change 
i

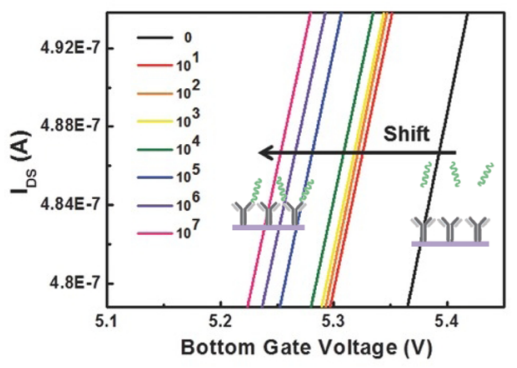

c i
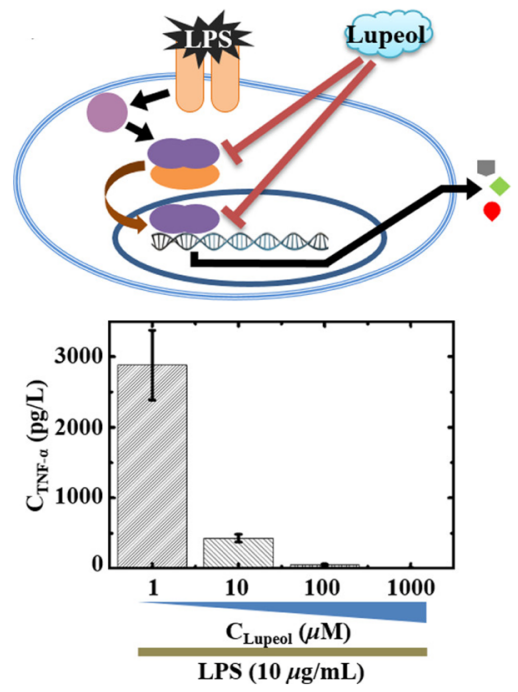

b

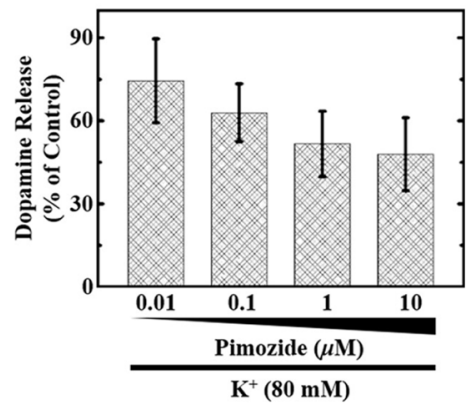

ii
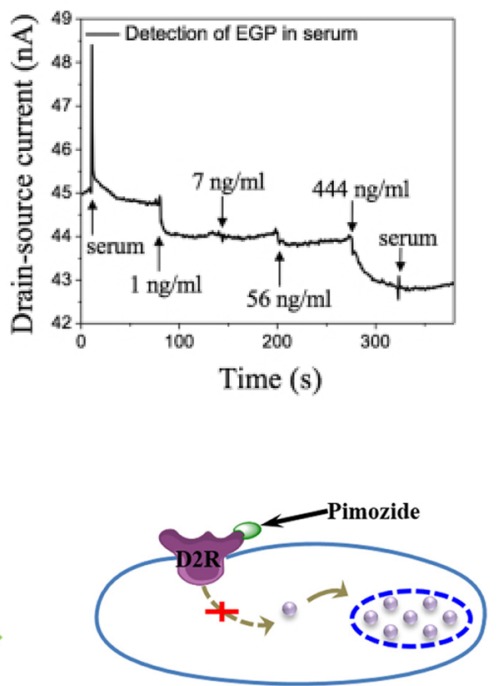

ii
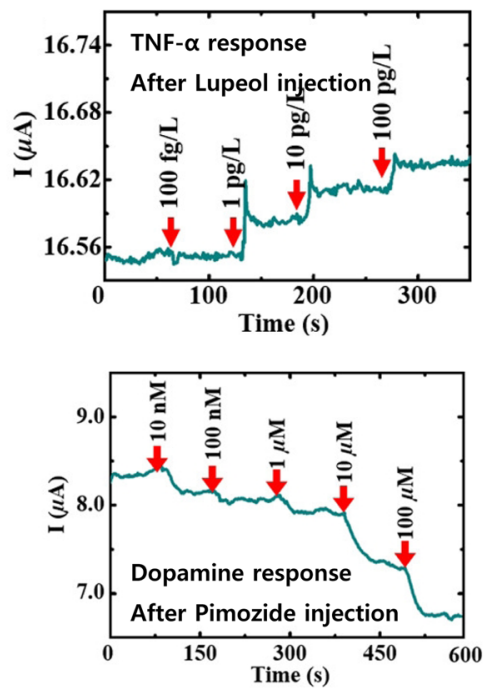

Fig. 5 Representative applications using BioFET. a Responses of FET sensors that can detect virus antigen directly. These detect avian influenza virus (AIV) and Ebola virus antigen through their nucleoprotein (NP) from a live animal cloacal swab and glycoprotein (GP) from human serum, with $\mathrm{I}_{\mathrm{ds}}$ change according to antigen concentration. (Reproduced with permission, 2017, Wiley and 2017, Nature Publishing Group). b Response of FET sensor that can detect biomarker of Alzheimer's disease, amyloid-beta 42 (A $\beta 42$ ). With reduced graphene oxide (rGO) and RNA aptamer in biomarker solution at $\mathrm{pH} 7.4$, the interaction between $\mathrm{A} \beta 42(\mathrm{pI}=5.5)$ and aptamer makes a shift of the transfer curve by inducing $\mathrm{p}$-doping to
FET. (Reproduced with permission, 2020, IEEE Sensors Council). c Specific cell signal transfer processes and measured figures that allows evaluating such blocking effect of drugs. Cell stimulating through lipopolysaccharides (LPS) and potassium make the release of tumor necrosis factor $\alpha(\mathrm{TNF}-\alpha)$ and dopamine each, which can be blocked by Lupeol and Pimozide. By using anti-TNF- $\alpha$ antibody and 2,2'-azino-bis (3-ethylbenzothiazoline-6-sulfonic acid) (ABTS•) radicals, the selective reaction between these and TNF- $\alpha$ / dopamine allows us to use FET sensors in the drug screening field. (Reproduced with permission, 2018, ACS and 2019, ACS)

\section{Declaration}

Conflict of interests The authors declare that they have no conflict of interest

\section{References}

Medical Device Development Fund (the Ministry of Science and ICT) (KMDF-202012D29). Authors especially thank to Seongbin Jo and Min Young Lee, who contributed proofreading and schematic drawing of this manuscript, respectively.
1. Schöning MJ, Poghossian A. Recent advances in biologically sensitive field-effect transistors (BioFETs). Analyst. 2002. https://doi. org/10.1039/b204444g. 
2. Gao A, Yang X, Tong J, et al. Multiplexed detection of lung cancer biomarkers in patients serum with CMOS-compatible silicon nanowire arrays. Biosens Bioelectron. 2016. https://doi. org/10.1016/j.bios.2016.12.072.

3. Veeralingam S, Badhulika S. Surface functionalized $\beta$-Bi2O3 nanofibers based flexible, field-effect transistor-biosensor (BioFET) for rapid, label-free detection of serotonin in biological fluids. Sens Actuators, B Chem. 2020. https://doi.org/ 10.1016/j.snb.2020.128540.

4. Stine R, Mulvaney SP, Robinson JT, Tamanaha CR, Sheehan PE. Fabrication, optimization, and use of graphene field effect sensors. Anal Chem. 2013. https://doi.org/10.1021/ac303190w.

5. Shin DH, Kim W, Jun J, Lee JS, Kim JH, Jang J. Highly selective FET-type glucose sensor based on shape-controlled palladium nanoflower-decorated graphene. Sensors Actuators, B Chem. 2018;264:216-23. https://doi.org/10.1016/j.snb.2018.02. 139.

6. He Q, Sudibya HG, Yin Z, et al. Centimeter-long and large-scale micropatterns of reduced graphene oxide films: fabrication and sensing applications. ACS Nano. 2010. https://doi.org/10.1021/ nn100780v.

7. Siddique S, Iqbal MZ, Mukhtar H. Cholesterol immobilization on chemical vapor deposition grown graphene nanosheets for biosensors and bioFETs with enhanced electrical performance. Sens Actuators, B Chem. 2017;253:559-65. https://doi.org/10.1016/j. snb.2017.06.170.

8. Tung NT, Tue PT, Thi Ngoc Lien T, et al. Peptide aptamer-modified single-walled carbon nanotube-based transistors for highperformance biosensors. Sci Rep. 2017;7(1):1-9. https://doi.org/ 10.1038/s41598-017-18169-1.

9. Lowe BM, Sun K, Zeimpekis I, Skylaris CK, Green NG. Fieldeffect sensors-from $\mathrm{pH}$ sensing to biosensing: sensitivity enhancement using streptavidin-biotin as a model system. Analyst. 2017. https://doi.org/10.1039/c7an00455a.

10. Koyappayil A, Lee MH. Ultrasensitive materials for electrochemical biosensor labels. Sensors. 2021. https://doi.org/10.3390/s2101 0089.

11. Liu S, Guo X. Carbon nanomaterials field-effect-transistor-based biosensors. NPG Asia Mater. 2012. https://doi.org/10.1038/am. 2012.42.

12. Ajayan PM. Nanotubes from Carbon. Chem Rev. 1999. https:// doi.org/10.1021/cr970102g.

13. Yang W, Ratinac KR, Ringer SR, Thordarson P, Gooding JJ, Braet F. Carbon nanomaterials in biosensors: should you use nanotubes or graphene. Angew Chemie - Int Ed. 2010. https://doi.org/10. 1002/anie.200903463.

14. Camilli L, Passacantando M. Advances on sensors based on carbon nanotubes. Chemosens. 2018. https://doi.org/10.3390/chemo sensors6040062.

15. Hwang MT, Heiranian M, Kim Y, et al. Ultrasensitive detection of nucleic acids using deformed graphene channel field effect biosensors. Nat Commun. 2020. https://doi.org/10.1038/ s41467-020-15330-9.

16. Alabsi SS, Ahmed AY, Dennis JO, Khir MHM, Algamili AS. A review of carbon nanotubes field effect-based biosensors. IEEE Access. 2020;8:69509-21. https://doi.org/10.1109/ACCESS. 2020.2987204

17. Jiang L, Santiago I, Foord J. Nanocarbon and nanodiamond for high performance phenolics sensing. Commun Chem. 2018. https://doi.org/10.1038/s42004-018-0045-8.

18. Bezzon VDN, Montanheiro TLA, De Menezes BRC, et al. Carbon nanostructure-based sensors: a brief review on recent advances. Adv Mater Sci Eng. 2019. https://doi.org/10.1155/2019/4293073.

19. Mansouri Majd S, Salimi A. Ultrasensitive flexible FET-type aptasensor for CA 125 cancer marker detection based on carboxylated multi-walled carbon nanotubes immobilized onto reduced graphene oxide film. Anal Chim Acta. 2018;1000:273-82. https:// doi.org/10.1016/j.aca.2017.11.008.

20. Seo G, Lee G, Kim MJ, et al. Rapid detection of covid-19 causative virus (SARS-CoV-2) in human nasopharyngeal swab specimens using field-effect transistor-based biosensor. ACS Nano. 2020;14(4):5135-42. https://doi.org/10.1021/acsnano.0c02823.

21. Liang Y, Xiao M, Wu D, et al. Wafer-scale uniform carbon nanotube transistors for ultrasensitive and label-free detection of disease biomarkers. ACS Nano. 2020;14(7):8866-74. https://doi.org/ 10.1021/acsnano.0c03523.

22. Yang H, Kim D, Kim J, et al. nanodisc-based bioelectronic nose using olfactory receptor produced in escherichia coli for the assessment of the death-associated odor cadaverine. ACS Nano. 2017. https://doi.org/10.1021/acsnano.7b04992.

23. Park C, Choi W, Kim D, Jin B, Lee JS. Highly sensitive detection of influenza a $(\mathrm{h} 1 \mathrm{n} 1)$ virus with silicon nanonet BioFETs. IEEE Sens J. 2019. https://doi.org/10.1109/JSEN.2019.2936216.

24. Meir R, Zverzhinetsky M, Harpak N, et al. Direct detection of uranyl in urine by dissociation from aptamer-modified nanosensor arrays. Anal Chem. 2020. https://doi.org/10.1021/acs.analchem. 0c02387.

25. Bhat KS, Ahmad R, Yoo JY, Hahn YB. Nozzle-jet printed flexible field-effect transistor biosensor for high performance glucose detection. J Colloid Interface Sci. 2017;506:188-96. https://doi. org/10.1016/j.jcis.2017.07.037.

26. Jung M, Kim J, Noh J, et al. All-Printed and roll-to-roll-printable 13.56-MHz operated 1-bit RF tag on plastic foils. IEEE Trans Electron Devices. 2010;57(3):571-80. https://doi.org/10.1109/ TED.2009.2039541.

27. Street RA, Wong WS, Ready SE, et al. Jet printing flexible displays. Mater Today. 2006. https://doi.org/10.1016/S13697021(06)71445-6.

28. Janissen R, Sahoo PK, Santos CA, et al. InP nanowire biosensor with tailored biofunctionalization: ultrasensitive and highly selective disease biomarker detection. Nano Lett. 2017. https://doi.org/ 10.1021/acs.nanolett.7b01803.

29. Patolsky F, Zheng G, Lieber CM. Fabrication of silicon nanowire devices for ultrasensitive, label-free, real-time detection of biological and chemical species. Nat Protoc. 2006. https://doi.org/10. 1038/nprot.2006.227.

30. Wang B, Luo Y, Gao L, Liu B, Duan G. High-performance field-effect transistor glucose biosensors based on bimetallic $\mathrm{Ni} / \mathrm{Cu}$ metal-organic frameworks. Biosens Bioelectron. 2020;2021(171):112736. https://doi.org/10.1016/j.bios.2020. 112736.

31. Mirsian S, Khodadadian A, Hedayati M, Manzour-ol-Ajdad A, Kalantarinejad R, Heitzinger C. A new method for selective functionalization of silicon nanowire sensors and bayesian inversion for its parameters. Biosens Bioelectron. 2019;142(April):111527. https://doi.org/10.1016/j.bios.2019.111527.

32. Manga YB, Ko FH, Yang YS, et al. Ultra-fast and sensitive silicon nanobelt field-effect transistor for high-throughput screening of alpha-fetoprotein. Sensors Actuators, B Chem. 2018;256:111421. https://doi.org/10.1016/j.snb.2017.10.007.

33. Lee HW, Kang DH, Cho JH, Lee S, Jun DH, Park JH. Highly sensitive and reusable membraneless field-effect transistor (fet)-type tungsten diselenide (WSe2) biosensors. ACS Appl Mater Interfaces. 2018;10(21):17639-45. https://doi.org/10.1021/acsami. $8 \mathrm{~b} 03432$.

34. Chae MS, Yoo YK, Kim J, Kim TG, Hwang KS. Graphene-based enzyme-modified field-effect transistor biosensor for monitoring drug effects in Alzheimer's disease treatment. Sensors Actuators, B Chem. 2018;272(June):448-58. https://doi.org/10.1016/j.snb. 2018.06.010.

35. Xu S, Jiang S, Zhang C, et al. Ultrasensitive label-free detection of DNA hybridization by sapphire-based graphene field-effect 
transistor biosensor. Appl Surf Sci. 2018;427:1114-9. https:// doi.org/10.1016/j.apsusc.2017.09.113.

36. Gong H, Chen F, Huang Z, et al. Biomembrane-modified field effect transistors for sensitive and quantitative detection of biological toxins and pathogens. ACS Nano. 2019;13(3):3714-22. https://doi.org/10.1021/acsnano.9b00911.

37. Kim W, Lee JS, Shin DH, Jang J. Platinum nanoparticles immobilized on polypyrrole nanofibers for non-enzyme oxalic acid sensor. J Mater Chem B. 2018;6(8):1272-8. https://doi.org/10.1039/c7tb0 0629b.

38. Stern E, Wagner R, Sigworth FJ, Breaker R, Fahmy TM, Reed MA. Importance of the debye screening length on nanowire field effect transistor sensors. Nano Lett. 2007. https://doi.org/10.1021/ nl071792z.

39. Vacic A, Criscione JM, Rajan NK, Stern E, Fahmy TM, Reed MA. Determination of molecular configuration by debye length modulation. J Am Chem Soc. 2011. https://doi.org/10.1021/ja205 684a.

40. Chu CH, Sarangadharan I, Regmi A, et al. Beyond the debye length in high ionic strength solution: direct protein detection with field-effect transistors (FETs) in human serum. Sci Rep . 2017. https://doi.org/10.1038/s41598-017-05426-6.

41. Bhattacharyya IM, Shalev G. Electrostatically governed debye screening length at the solution-solid interface for biosensing applications. ACS Sensors. 2020. https://doi.org/10.1021/acsse nsors.9b01939.

42. Welch NG, Scoble JA, Muir BW, Pigram PJ. Orientation and characterization of immobilized antibodies for improved immunoassays (Review). Biointerphases. 2017. https://doi.org/10.1116/1. 4978435.

43. Rashid JIA, Yusof NA. The strategies of DNA immobilization and hybridization detection mechanism in the construction of electrochemical DNA sensor: A review. Sens Bio-Sensing Res. 2017. https://doi.org/10.1016/j.sbsr.2017.09.001.

44. Nimse SB, Song K, Sonawane MD, Sayyed DR, Kim T. Immobilization techniques for microarray: challenges and applications. Sensors . 2014. https://doi.org/10.3390/s141222208.
45. Park S, Choi J, Jeun M, et al. Detection of avian influenza virus from cloacal swabs using a disposable well gate fet sensor. Adv Healthc Mater. 2017;6(13):1-6. https://doi.org/10.1002/adhm. 201700371.

46. Chen Y, Ren R, Pu H, et al. Field-effect transistor biosensor for rapid detection of ebola antigen. Sci Rep. 2017;7(1):4-11. https:// doi.org/10.1038/s41598-017-11387-7.

47. Salehirozveh M, Dehghani P, Zimmermann M, Roy VAL, Heidari $\mathrm{H}$. Graphene field effect transistor biosensors based on aptamer for amyloid- $\beta$ detection. IEEE Sens J. 2020;20(21):12488-94. https:// doi.org/10.1109/JSEN.2020.3000583.

48. Pham Ba VA, Han YM, Cho Y, et al. Modified floating electrodebased sensors for the quantitative monitoring of drug effects on cytokine levels related with inflammatory bowel diseases. ACS Appl Mater Interfaces. 2018;10(20):17100-6. https://doi.org/10. 1021/acsami.8b04287.

49. Pham Ba VA, Cho DG, Hong S. Nafion-radical hybrid films on carbon nanotube transistors for monitoring antipsychotic drug effects on stimulated dopamine release. ACS Appl Mater Interfaces. 2019;11(10):9716-23. https://doi.org/10.1021/acsami. $8 b 18752$.

50. Rajan NK, Brower K, Duan X, Reed MA. Limit of detection of field effect transistor biosensors: effects of surface modification and size dependence. Appl Phys Lett. 2014. https://doi.org/10. 1063/1.4867025.

51. Xu JJ, Luo XL, Chen HY. Analytical aspects of FET-based biosensors. Front Biosci. 2005. https://doi.org/10.2741/1538.

Publisher's Note Springer Nature remains neutral with regard to jurisdictional claims in published maps and institutional affiliations. 